\title{
CD146/MCAM defines functionality of human bone marrow stromal stem cell populations
}

\author{
Linda Harkness $^{1^{*}} \mathbb{D}$, Walid Zaher ${ }^{1,3}$, Nicholas Ditzel ${ }^{1}$, Adiba Isa ${ }^{1}$ and Moustapha Kassem,2,3
}

\begin{abstract}
Background: Identification of surface markers for prospective isolation of functionally homogenous populations of human skeletal (stromal, mesenchymal) stem cells (hMSCs) is highly relevant for cell therapy protocols. Thus, we examined the possible use of CD146 to subtype a heterogeneous hMSC population.

Methods: Using flow cytometry and cell sorting, we isolated two distinct hMSC-CD146 ${ }^{+}$and hMSC-CD146 cell populations from the telomerized human bone marrow-derived stromal cell line (hMSC-TERT). Cells were examined for differences in their size, shape and texture by using high-content analysis and additionally for their ability to differentiate toward osteogenesis in vitro and form bone in vivo, and their migrational ability in vivo and in vitro was investigated.

Results: In vitro, the two cell populations exhibited similar growth rate and differentiation capacity to osteoblasts and adipocytes on the basis of gene expression and protein production of lineage-specific markers. In vivo, hMSCCD146 ${ }^{+}$and hMSC-CD146 ${ }^{-}$cells formed bone and bone marrow organ when implanted subcutaneously in immune-deficient mice. Bone was enriched in hMSC-CD146- cells (12.6 \% versus $8.1 \%$ ) and bone marrow elements enriched in implants containing hMSC-CD $146^{+}$cells (0.5 \% versus $\left.0.05 \%\right)$. hMSC-CD $146^{+}$cells exhibited greater chemotactic attraction in a transwell migration assay and, when injected intravenously into immune-deficient mice following closed femoral fracture, exhibited wider tissue distribution and significantly increased migration ability as demonstrated by bioluminescence imaging.

Conclusion: Our studies demonstrate that CD146 defines a subpopulation of hMSCs capable of bone formation and in vivo trans-endothelial migration and thus represents a population of hMSCs suitable for use in clinical protocols of bone tissue regeneration.
\end{abstract}

Keywords: Bone marrow stem cells, CD146/MCAM, Osteogenic differentiation, Bone formation

\section{Background}

Human bone marrow (BM) skeletal stem cells (also termed marrow stromal cells or mesenchymal stem cells) (hMSCs) are multipotent stem cells present in the BM stroma and are capable of mesoderm lineage differentiation (e.g., osteoblasts, adipocytes, and chondrocytes) [1]. hMSCs are being tested for treatment in a number of degenerative diseases, including bone $[2,3]$ and cartilage [4] regeneration. hMSCs isolated and expanded under current culture conditions

\footnotetext{
*Correspondence: Iharkness@health.sdu.dk

'Department of Endocrinology and Metabolism, Molecular Endocrinology Laboratory (KMEB), Odense University Hospital, University of Southern Denmark, Winslowparken 25.1, 5000 Odense C, Denmark

Full list of author information is available at the end of the article
}

that rely on plastic adherence are heterogeneous with respect to differentiation. Single-cell cloning of hMSCs revealed that approximately $20-40 \%$ of the cell population are multipotent MSCs and the remaining are probably cells at different stages of differentiation $[5,6]$. In addition, Russell et al. reported that only $50 \%$ of clonal BM hMSCs exhibited tri-lineage differentiation (osteoblasts, adipocytes, and chondrocytes) [7]. We recently reported that canonical osteoblastic markers (e.g., alkaline phosphate and osteocalcin) are not predictive for in vivo bone-forming capacity of hMSCs or hMSC "stemness" [8] and that there exist, in MSC cultures, cell populations committed to adipocyte or osteoblast lineages [9]. More recently, lineage-tracing 
studies in vivo have corroborated the presence of heterogeneity within the MSC population [10]. This functional heterogeneity of hMSCs limits the clinical use of MSCs in therapy and may explain the varied results obtained from clinical trials $[11,12]$. Thus, one of the challenges facing the use of hMSCs in therapy is the identification of prospective markers that predict their in vivo functionality.

A number of studies have isolated and characterized distinct populations of BM hMSCs by using a number of surface markers (e.g., Stro-1 and CD105 [13], CD271 [14], and CD56 [15, 16], and alkaline phosphatase (ALP) [17]). Although these markers enrich for an hMSC population with trilineage differentiation and colony-forming abilities, the isolated cells were still heterogeneous with respect to differentiation potential.

Cluster of differentiation 146 (CD146), also known as melanoma cell adhesion molecule (MCAM, MelCAM) or cell surface glycoprotein Muc18, was originally identified as an endothelial cell marker with a role in cell-matrix interaction and angiogenesis. CD146 defines the self-renewing hMSC population located in perivascular space in BM [18]. Additionally, CD146 expression has been reported to be higher in hMSC multipotent clones compared with hMSC unipotent clones [7] and to be correlated with osteoblastic differentiation potential $[18,19]$. Conversely, Tormin et al. [14] reported that multipotent hMSCs are present in both the $\mathrm{CD} 146^{-}$and $\mathrm{CD} 146^{+}$populations and that these populations exist within two different niches in vivo: endosteal and perivascular. Thus, differences and similarities of the $\mathrm{CD} 146^{-}$and $\mathrm{CD} 146^{+}$populations of hMSCs have not been totally clarified.

We have previously established a human telomeraseoverexpressing hMSC line (termed hMSC-TERT [20]) that maintains "stemness" characteristics of hMSCs in spite of extensive in vitro proliferation. hMSC-TERT exhibit a mixed expression of CD146 and thus provided us with the opportunity to characterize, in a prospective fashion, the phenotype of hMSCs defined by CD146 expression. Here, we compare the biological characteristics of $\mathrm{CD}_{146^{+}}$and $\mathrm{CD} 146^{-}$cell populations by employing in vitro and in vivo assays.

\section{Methods}

\section{Cell cultures}

We employed the parental telomerised cell line hMSCTERT (subclone hMSC-TERT4) described previously [20]. To visualize the cells when implanted in vivo, we created a sub-line from hMSC-TERT4, overexpressing firefly luciferase (LUC2) and termed hMSC-LUC2.

\section{Stable retroviral transfection using the firefly luciferase gene LUC2}

The LUC2 gene was polymerase chain reaction (PCR)amplified from the pGL4.10 plasmid by using Phusion Hot
Start High-Fidelity DNA Polymerase (Thermo Fisher Scientific, Waltham, MA, USA) in accordance with the instructions of the manufacturer. The primers sequences were as follows: forward primer: CATCAGCCAGCCCACCGTCG and reverse primer: CGGTCGAAGCTCTCGGGCAC.

The PCR products were purified with the SV gel and PCR clean-up system (Promega, Nacka, Sweden) and ligated into the retroviral vector pBABEpuro, which was pre-digested with the SnaBI (blunt) restriction enzyme. The plasmid was then transformed into DH5 $\alpha$ cells. Positive clones were identified by purifying the vector by using a Wizard Plus SV Minipreps DNA Purification System (Promega) in accordance with the instructions of the manufacturer. After purification, the plasmid was linearized and analyzed on a $0.5 \%$ agarose gel for $90 \mathrm{~min}$ at $70 \mathrm{~V}$. Finally, the inserts were confirmed by sequencing.

\section{Virus generation and transfection}

Phoenix A packaging cells (70-80 \% confluent), cultured in six-well plates, were transfected with the pBABE-LUC2 construct ( $3 \mu \mathrm{g} / \mathrm{well})$ by using the FuGENE 6 (Roche, Hvidovre, Denmark) method in accordance with the instructions of the manufacturer. The supernatants, from $25 \mathrm{~cm}^{2}$ of Phoenix A packaging cells containing virus particles, were collected 24 and $48 \mathrm{~h}$ after transfection, filtered with a $0.45-\mu \mathrm{m}$ filter, diluted 1:1 with the culture medium, and added to the hMSC-TERT cell line, in a $25-\mathrm{cm}^{2}$ flask supplemented with $6 \mu \mathrm{g} / \mathrm{ml}$ Polybrene for infection. Twentyfour hours after the second round of infection, $3 \mu \mathrm{g} / \mathrm{ml}$ puromycin was added for selection, and the selection pressure was maintained until all non-transfected control cells were eradicated. The puromycin-resistant cells were expanded and maintained in medium supplemented with $0.2 \mu \mathrm{g} / \mathrm{ml}$ puromycin. An estimated 500,000 cells initially survived the selection to make the respective cell line (termed hMSC-LUC2).

\section{CD146 cell sorting}

The hMSC-LUC2, was used for all experiments. hMSCLUC2 cells were sorted on a FACSdiva (BD Biosciences, Brøndby, Denmark) into positive (hMSC-CD146 ${ }^{+}$) and negative (hMSC-CD146-) cell populations using incubation with a PE pre-conjugated CD146 antibody (BD Pharmingen, Brøndby, Denmark). Briefly, hMSC-LUC2 cells were trypsinized to a single-cell suspension, washed in phosphate-buffered saline (PBS) with $0.5 \%$ bovine serum albumin (BSA), and incubated with CD146 antibody diluted in PBS with $0.5 \%$ BSA for 30 min on ice. Cells were then sorted into distinct populations on a FACSAria cell sorter (BD Biosciences) and re-cultured for future in vitro and in vivo experiments. Cell expansion was performed in basal media (minimum essential medium) (Invitrogen, Taastrup, Denmark with $10 \%$ fetal bovine serum (FBS); PAA, Pasching, Austria). 


\section{Cell proliferation}

Cell proliferation was monitored by determining the number of population doublings by using the formula: $\log \mathrm{N} / \log 2$, where $\mathrm{N}$ is the cell number of the confluent monolayer divided by the initial number of seeded cells.

\section{Cell differentiation}

For osteoblast differentiation, the cells were cultured in osteoblastic induction media (OIM) comprised of basal media supplemented with $10 \mathrm{mM} \beta$-glycerophosphate (Calbiochem-Merck, Darmstadt, Germany), $50 \mu \mathrm{g} / \mathrm{ml} \mathrm{L-}$ ascorbic acid-2-phosphate (Wako Chemicals $\mathrm{GmbH}$, Neuss, Germany), $10 \mathrm{nM}$ dexamethasone (Sigma-Aldrich, Brøndby, Denmark), and $10 \mathrm{nM}$ calcitriol (1.25-dihydroxy vitamin- $\mathrm{D}_{3}\left(1,25(\mathrm{OH})_{2} \mathrm{D}_{3}\right)$ kindly provided by Leo Pharma, Ballerup, Denmark). For adipocyte differentiation, the cells were cultured in adipocytic induction media (AIM) containing basal media supplemented with $10 \%$ horse serum (Sigma-Aldrich), $100 \mathrm{nM}$ dexamethasone (Sigma-Aldrich), $500 \mu \mathrm{M}$ 1-methyl-3-isobutylxanthine (IBMX) (Sigma-Aldrich), $1 \mu \mathrm{M}$ Rosiglitazone (BRL49653; Cayman Chemical, Ann Arbor, MI, USA), and $5 \mu \mathrm{g} / \mathrm{ml}$ insulin (Sigma-Aldrich). Samples undergoing induction were collected at days 5,10 , and 15 . Three independent experiments were performed for each differentiation assay.

\section{Flow cytometry}

Flow cytometry was performed by using a FACScan (BD Biosciences). To confirm the profile of either hMSC-TERT versus hMSC-LUC2 or hMSC-CD146 ${ }^{+}$and hMSC-CD146 populations, cells were trypsinized to a single-cell suspension, washed in PBS $+0.5 \% \mathrm{BSA}$, and incubated with an antibody (in PBS + $0.5 \%$ BSA) for $30 \mathrm{~min}$ on ice. After incubation, excess antibody was washed out by using PBS and cells analyzed on the FACSCalibur (BD Biosciences) flow cytometer and data analyzed by using WinMDI (The Scripps Institute, Flow Cytometry Core Facility). Sorted and unsorted cell populations were profiled using a number of known MSC pre-conjugated fluorescence-activated cell sorting (FACS) markers: CD14-FITC, CD34-PE, CD44-PE, CD63-FITC, CD73-PE, and CD146-PE (all BD Pharmingen) and CD105-APC (eBioscience, Hatfield, UK).

\section{Alkaline phosphatase activity and cell viability}

Cell viability was measured by using CellTiter-Blue Cell Viability assay in accordance with the instructions of the manufacturer (Promega). ALP activity was determined by using a $1 \mathrm{mg} / \mathrm{ml}$ solution of P-nitrophenylphosphate (Sigma-Aldrich) in $50 \mathrm{mM} \mathrm{NaHCO} 3$ with $1 \mathrm{mM} \mathrm{MgCL}_{2}$, $\mathrm{pH}$ 9.6, at $37{ }^{\circ} \mathrm{C}$ for $20 \mathrm{~min}$; activity was then stopped with $3 \mathrm{M} \mathrm{NaOH}$. Plates were read by using a FLUOstar Omega plate reader (BMG Laboratories, Ramcon A/S,
Birkerod, Denmark), and ALP activity was corrected for cell number.

\section{Cytochemical staining}

Cells undergoing osteoblastic or adipocytic induction were stained at days 5, 10, and 15 for ALP and alizarin red or Oil red $\mathrm{O}(\mathrm{ORO})$, respectively, as previously described [21]. Elution of ORO staining was performed by using $100 \mu \mathrm{l}$ of isopropanol for $10 \mathrm{~min}$ at room temperature; $60 \mu \mathrm{l}$ was then removed to a 96 -well plate and read on a FLUOstar Omega plate reader set at $500 \mathrm{~nm}$ emission wavelength.

\section{Cell morphology analysis by high-content imaging} hMSC-CD146 ${ }^{+}$and hMSC-CD146 $6^{-}$cells were seeded in 96-cell carrier well plates (PerkinElmer, Waltham, MA, USA) and cultured in standard medium. The adherent cells were washed with $\mathrm{PBS}^{-/-}$, fixed in $4 \%$ formaldehyde for $10 \mathrm{~min}$ at room temperature, and washed three times with PBS. Cells were stained for alpha tubulin, Phalloidin-TRITC, and Hoechst 33342 (all SigmaAldrich) by using Alexafluor 488 (Thermo Fisher Scientific) as the secondary antibody for alpha tubulin. Plates were scanned in the Operetta (PerkinElmer) and cells $(>1000$ per cell line) analyzed for cell size and shape and changes in microtubule (F-actin) thickness using the Harmony software (PerkinElmer) and texture analysis using SER (saddle, edge, ridge) features to measure changes in structure within the cell. Both the 'Valley' and 'Ridge' patterns from the SER features were selected by using a 2px width to assess spaces or ridge height of the F-actin fibres.

\section{Boyden chamber transwell migration assay}

The migration ability of sorted cells was measured in a 48-well micro-chemotaxis Boyden chamber-based cell migration assay $(8-\mu \mathrm{m}$ pore polycarbonate membrane; Neuro Probe, Inc., Gaithersburg, MD, USA). The membrane was coated for $1 \mathrm{~h}$ at $37^{\circ} \mathrm{C}$ in migration media-low glucose Dulbecco's modified Eagle's medium (Invitrogen), with $0.2 \%$ FBS (Sigma-Aldrich)-supplemented with $5 \mu \mathrm{g} / \mathrm{ml}$ Fibronectin and $10 \mu \mathrm{g} / \mathrm{ml}$ rat-tail collagen I. The cells were cultured in migration media for $24 \mathrm{~h}$ prior to migration initiation. The chemotaxis and control media $(27 \mu \mathrm{l})$ were placed in the lower chamber. The chamber was covered by the coated membrane and fixed by the upper chamber. Fifty-microliter sorted cells $\left(2.5 \times 10^{5}\right.$ cells $/ \mathrm{ml}$ migration media) were loaded into the upper chamber wells and incubated for $4 \mathrm{~h}$ at $37^{\circ} \mathrm{C}$. Non-migrated cells on the upper side of the membrane were scraped and rinsed with cold PBS. Migrated cells on the lower side of the membrane were fixed and stained with a hemacolor staining kit (Merck) and imaged on an inverted microscope ( $\times 20$ magnification). Images were captured of the 
entire wells, and cells were counted by using the ImageJ program. Results were expressed as mean number of cells for six technical replicates ( $\mathrm{n}=2$ independent experiments).

\section{Gene expression analysis}

Total RNA was extracted from samples collected at days $0,5,10$, and 15 of induction from unsorted and sorted populations by using TRIzol (Invitrogen) in accordance with the instructions of the manufacturer. cDNA was synthesized by using a revertAid $\mathrm{H}$ minus first-strand cDNA synthesis kit (Fermentas, St Leon-Rot, Germany). Reverse transcription-polymerase chain reaction (RTPCR) was performed on an ABI StepOne ${ }^{\mathrm{rm}}$ Real-Time PCR machine (Life Technologies/Applied Biosystems), and data analyzed by using Excel (Microsoft Corporation, Redmond, WA, USA). Ten micrograms of cDNA was used in each reaction in combination with $2 \times$ Fast SYBR green master mix (Applied Biosystems). Primers were purchased from DNA Technology A/S (Risskov, Denmark) or Eurofins (Ebersberg, Germany) and included Collagen 1a1 (COL1a1), RUNX2, alkaline phosphatase (ALPL), osteocalcin (BGLAP), osteonectin (SPARC), osteopontin (OPN), the long and short forms of CD146 (lgCD146, shCD146), $C D 146$, elastin $(E L N)$, decorin $(D C N)$, and biglycan $(B G N)$ for osteogenic differentiation and adipocyte-specific lipid binding protein $2(a P 2), P P A R \gamma 2$, lipoprotein lipase $(L P L)$, adiponectin $(A D I P O Q)$, and $C / C E B P \alpha$ for adipogenic differentiation. Cycle threshold $(\mathrm{CT})$ values were normalized against $\beta$-actin and analysis was performed by using the comparative CT method. Statistical analysis to detect differences between gene expression levels was carried out by using Student's $t$ test. Primers used in these studies can be found in Additional file 1: Table S1.

\section{In vivo heterotopic bone formation assay}

The cells were trypsinized to a single-cell suspension and seeded onto $40 \mathrm{mg}$ Triosite (HA/TCP, 0.5- to 1-mm granules; Biomatlante/Zimmer, Vigneux de Bretagne, France) and kept overnight at $37{ }^{\circ} \mathrm{C}, 5 \% \mathrm{CO}_{2}$. HA/TCP granules in combination with cells were then implanted subcutaneously (four implants per cell line) in the dorso-lateral area of immune-compromised (NOD.CB17-Prkdc scid/J) mice (The Jackson Laboratory, Bar Harbor, ME, USA) for 8 weeks. Ethical approval for subcutaneous implantations was granted by the Danish National Animal Experiment Inspectorate (2012-DY-2934-00006). The implants were removed, fixed in formalin, decalcified using formic acid for 3 days or $0.4 \mathrm{M}$ ethylenediaminetetraacetic acid (EDTA) for 12 days, embedded, and sectioned. Four serial sections were cut at three different tissue depths with $100 \mu \mathrm{m}$ distance between each group. Staining was performed with hematoxylin and eosin (three sections) or human-specific Vimentin (\#RM-9120, Clone SP20; Thermo Fisher Scientific: one section). Additional staining was performed by using tartrate-resistant acid phosphate (TRAP) staining on implants which underwent $0.4 \mathrm{M}$ EDTA decalcification. In brief, $50 \mathrm{ml}$ acetate buffer $\mathrm{pH} 5.2$ with $115 \mathrm{mg}$ sodium tartrate and $70 \mathrm{mg}$ Fast Red Salt TR (Sigma-Aldrich, F2768) was mixed with $70 \mathrm{mg}$ napthol ASTR phosphate (Sigma-Aldrich, N6125) dissolved in $250 \mu \mathrm{l} \mathrm{N}$-N-dimethylformamide to produce the staining solution. The slides with tissue sections were deparaffinized in xylene and processed through increasing alcohol concentrations before being incubated in the staining solution for $2 \mathrm{~h}$ at $37{ }^{\circ} \mathrm{C}$. Sections were briefly counterstained in Mayer's hematoxylin before rinsing in tap water and mounting under coverslips with an aqueous mounting medium.

\section{Quantification of heterotopic bone formation and bone marrow}

Mosaic images of serial sections were obtained by using Surveyor (Objective Imaging, Cambridge, UK). Using the free-hand contouring tool from PhotoShop (Adobe, CS4), the tissue area of each implant was calculated. Using the Magic wand-tool, the bone or BM areas were highlighted and the areas calculated; data were then calculated as a percentage of bone or BM/tissue area of the implant (nine sections per implant).

\section{In vivo migration assay}

A closed fracture was created in 10 immunodeficient

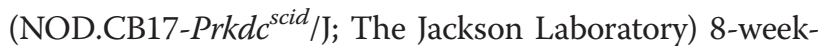
old, sedated, female mice $(100 \mathrm{mg} / \mathrm{kg}$ ketamine hydrochloride and $5 \mathrm{mg} / \mathrm{kg}$ xylazine hydrochloride) as described by Bonnarens and Einhorn [22]. Ethical approval for femoral fracture was granted by the Danish National Animal Experiment Inspectorate (2012-15-2934-00559). Fractures were created by using a guillotine apparatus on the right hind limb following insertion of a $0.5-\mathrm{mm}$ needle. Fracture and pinning were confirmed by $\mathrm{x}$-ray (Faxitron MX-20). Three hours after fracture, single-cell suspended hMSC$\mathrm{CD} 146^{+}$or hMSC-CD146 ${ }^{-}$cells were tail vein-injected at $1 \times 10^{6}$ cells per $100 \mu \mathrm{l}$ of PBS/injection into five mice per cell line and bioluminescence imaged at $30 \mathrm{~min}$ postinjection (d0), d1, d3, and d6.

\section{Bioluminescence imaging}

The Xenogen IVIS Spectrum (PerkinElmer) was used to obtain images of bioluminescence. Ten to 15 minutes prior to imaging, immunodeficient mice, tail vein-injected with either hMSC-CD146 ${ }^{+}$or hMSC-CD146 ${ }^{-}$cells, were injected intraperitoneally with D-luciferin (PerkinElmer, $150 \mathrm{mg} / \mathrm{kg}$ body weight in sterile PBS without $\mathrm{Mg}^{2+}$ and $\mathrm{Ca}^{2+}$ ) and then sedated with $2.5 \%$ isoflurane in oxygen. Images were obtained with field-of-view $13.2 \mathrm{~cm}$, binning factor $8, \mathrm{~F} / \mathrm{Stop} 1$, and automatic exposure time by using Xenogen Living Image ${ }^{\mathrm{Tm}}$ software (version 2.11) overlay 
(PerkinElmer). Radiance was plotted by using calibrated measurements of photon emission and displayed in units of photons $/$ second $/ \mathrm{cm}^{2} /$ steradian.

\section{Statistical analysis}

Three independent in vitro experiments were performed, and data were presented as mean \pm standard deviation. Statistical analysis were performed by using a one-way analysis of variance where group size was 3 or more, and for group sizes of 2, Student's $t$ test was applied $\left({ }^{*} P<0.05\right.$, ** $P<0.005$ or $P<0.01$, *** $P<0.0001$ ).

\section{Results}

\section{Characterization of hMSC-LUC2 cell line}

hTERT-LUC2 exhibited cell morphology (Additional file 2: Figure $\mathrm{S} 1 \mathrm{~A}$ ) and $\mathrm{CD}$ marker expression profile (Additional file 2: Figure S1C) similar to hMSC-TERT. However, hMSC-LUC2 demonstrated lowered proliferation rate compared with hMSC-TERT: population doubling per day was $0.45 \pm 0.06$ versus $0.58 \pm 0.04$, respectively $(P<0.005)$ (Additional file 2: Figure S1B). Ex vivo differentiation studies demonstrated that hTERTLUC2 maintained their osteoblastic (Additional file 3: Figure S2A) and adipocytic (Additional file 3: Figure S2B) differentiation capacities. RT-PCR data demonstrated similar gene expression profiles between the hMSC-TERT and hTERT-LUC2. Similar to hMSC-TERT, hMSC-LUC2 formed heterotopic bone and BM when implanted subcutaneously in immune-deficient mice (Additional file 4: Figure S3).

\section{Isolation and phenotypic characterization of hMSC-CD146 ${ }^{+}$and hMSC-CD146- cells}

hMSC-LUC2 cells $\left(18 \times 10^{6}\right)$ were sorted on the basis of the expression of CD146. Two distinct areas were gated (P4: hMSC-CD146 ${ }^{+}$and P5: hMSC-CD146 ${ }^{-}$; Fig. 1a), and cells remaining within the intermediary area $(40 \%$ of the total population) were discarded. After sorting of the

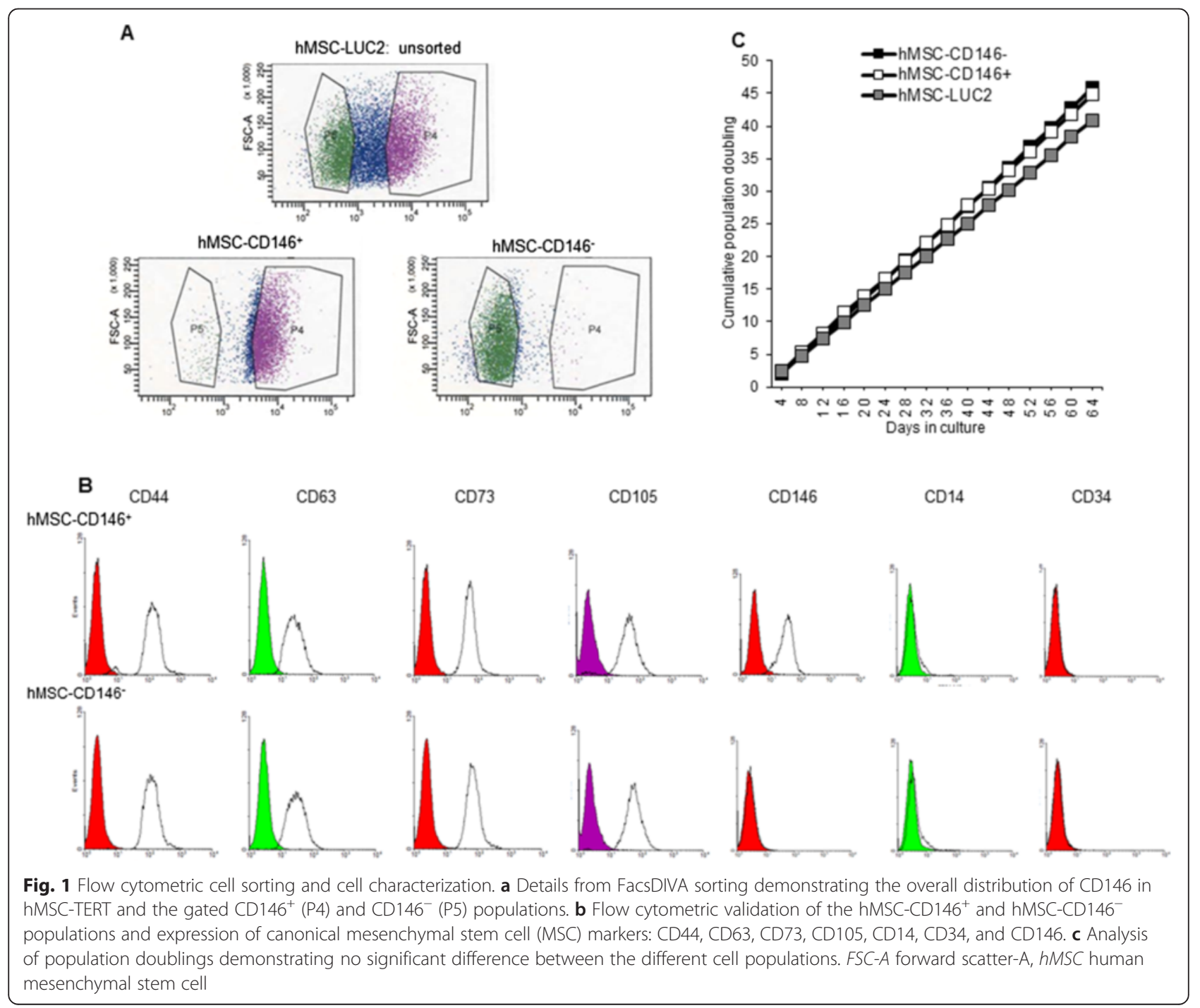


hMSC-CD $146^{+}$cells, $75.5 \%$ were gated within P4 and $89.1 \%$ of cells were gated within P5 (hMSC-CD146 ${ }^{-}$) (Fig. 1a). Immediately after cell sorting and for subsequent passages when cells were used for experimental purposes, flow cytometry was performed to confirm the CD146 status in both cell populations. The cells maintained their CD146 + or $\mathrm{CD} 146^{-}$status for six passages. In addition, the MSC identity of the cells was maintained as shown by the expression of a selected number of CD markers by FACS analysis: CD14 and CD34 (both negative) and CD44, CD63, CD73, and CD105 (all positive). We observed similar expression levels of these markers in hMSC-CD $146^{+}$and hMSC$\mathrm{CD}_{146}{ }^{-}$(Fig. 1b). Growth rate was identical between hMSC-LUC2, hMSC-CD146 ${ }^{+}$and hMSC-CD146 (Fig. 1c).

Quantitative cell morphology using high-content imaging hMSC-CD $146^{+}$and hMSC-CD $146^{-}$were examined for their differences in cell morphology and texture using high-content imaging analysis. Significant differences in cell area, roundness, and width-to-length ratio were observed between hMSC-CD $146^{+}$and hMSC-CD146 (Fig. 2). hMSC-CD $146^{+}$were rounder than hMSCCD146 ${ }^{-}$cells and demonstrated a smaller cytoplasmic area. Analysis of the F-actin filaments (stained with Phalloidin) using SER texture analysis demonstrated that the hMSC-CD $146^{-}$cells contained significantly larger fibres than hMSC-CD146 ${ }^{+}(P<0.05$; Fig. 2$)$.

\section{Osteoblast differentiation of hMSC-CD $146^{+}$and hMSC- CD146-}

No consistent significant differences between gene expression levels in hMSC-CD $146^{+}$and hMSC-CD146- were observed (Fig. 3a). Similarly, no differences were observed between hMSC-CD $146^{+}$and hMSC-CD $146^{-}$in ALP activity (Fig. 2b) or ALP cytochemical staining (Fig. 3c) or in their ability to form mineralized matrix as visualized by

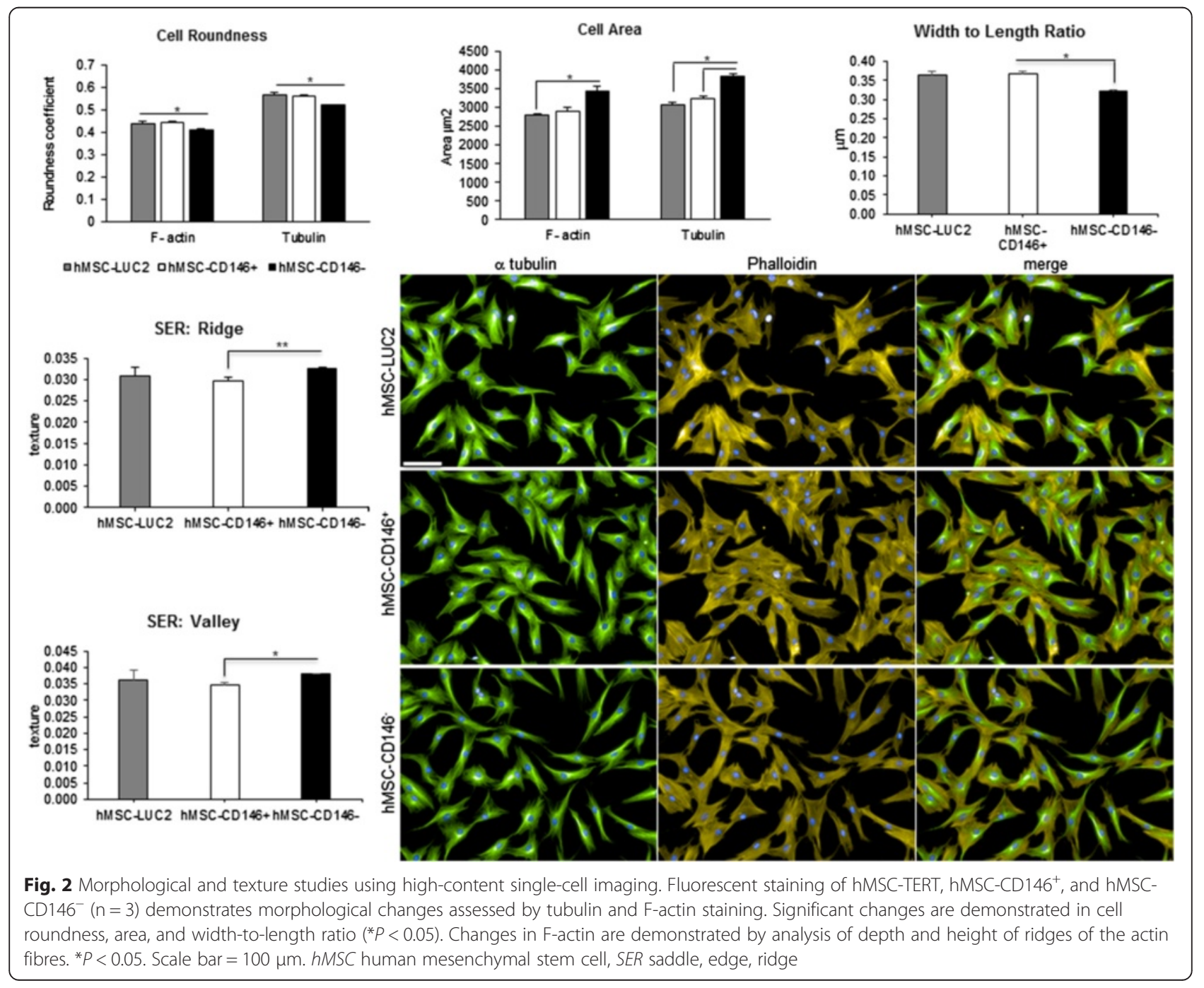




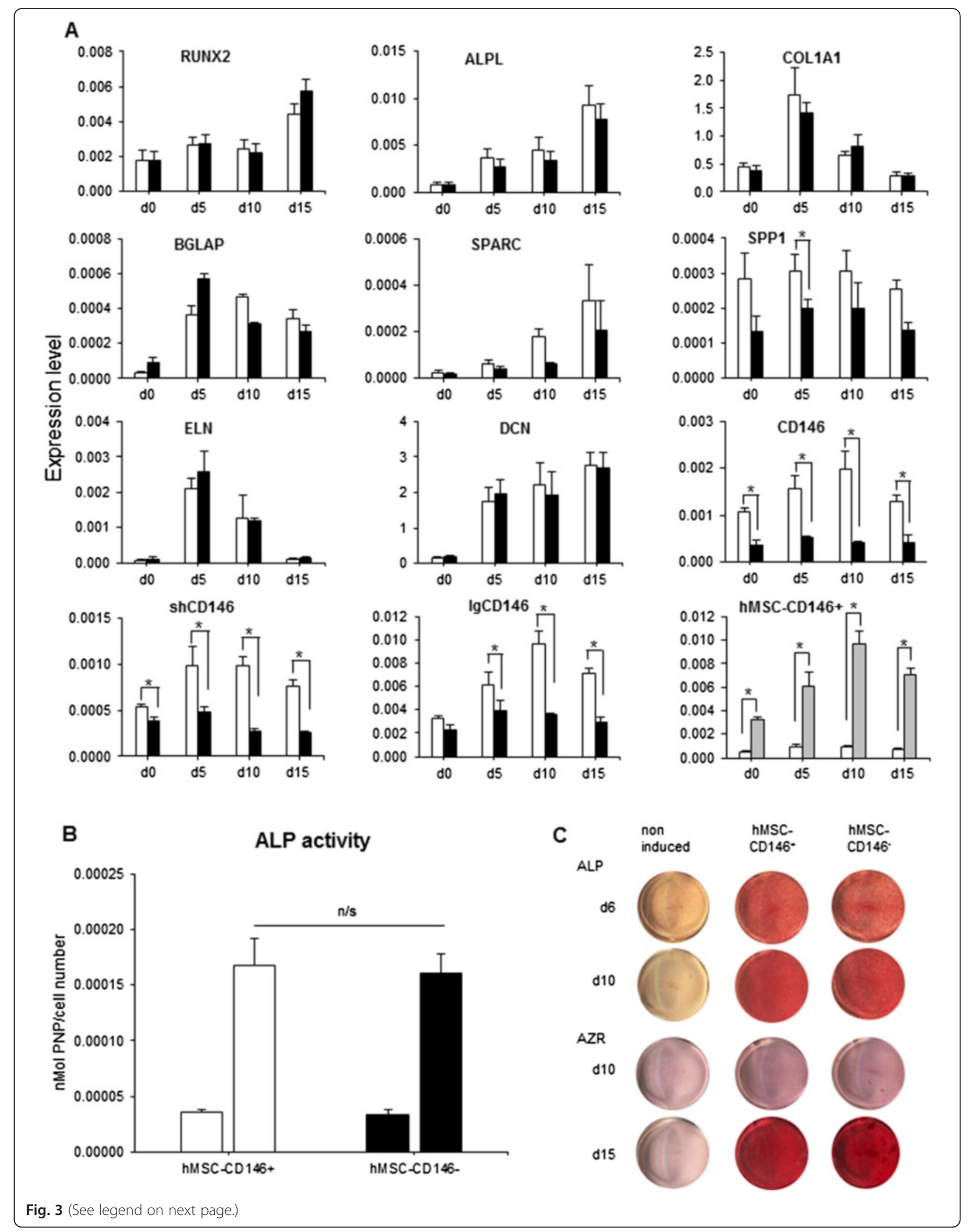


(See figure on previous page.)

Fig. 3 Characterization of sorted CD146 populations undergoing osteoblast (OB) differentiation. a Reverse transcription-polymerase chain reaction data (mean \pm standard error of the mean, $n=3$ independent experiments) analyzed from sorted population at day (d) $0,5,10$, and $15 .{ }^{*} P<0.05$. $\mathrm{sh}=$ short form and $\mathrm{lg}=$ long form of CD146. $\mathbf{b}$ Alkaline phosphatase (ALP) activity/cell viability at $\mathrm{d} 6$ of osteoblastic differentiation. $\mathbf{c}$ ALP staining at $\mathrm{d} 6$ and $\mathrm{d} 10$ of $\mathrm{OB}$ differentiation. Alizarin red (AZR) staining at $\mathrm{d} 10$ and $\mathrm{d} 15$ of $\mathrm{OB}$ differentiation. $\mathrm{n}=3$ independent experiments. white box, hMSC-CD146'; black box, hMSC-CD146-; gray box, lgCD146+

alizarin red staining (Fig. 3c). The phenotype of hMSCCD146 ${ }^{+}$and hMSC-CD146 ${ }^{-}$was stable during osteoblast differentiation as demonstrated by gene expression levels of CD146 isoforms (Fig. 3a).

CD146 was previously reported to have two isoforms-short and long, generated by alternative splicing of the cytoplasmic region-which probably mediate different functions [23]. We found that the long form of CD146 (lgCD146), but not the short form (shCD146), expression levels were upregulated during $\mathrm{OB}$ differentiation $(P<0.05)$ (Fig. 3a).

\section{Adipocytic differentiation of hMSC-CD146 ${ }^{+}$and hMSC- CD146-}

Both hMSC-CD146 ${ }^{+}$and hMSC-CD146 ${ }^{-}$exhibited similar differentiation capacity to adipocytes as revealed by comparable gene expression levels of adipocytic differentiation markers: peroxisome proliferator-activated receptor gamma 2 (PPARy2), lipoprotein Lipase ( $L P L)$, fatty acid binding protein 4 (FABP4/aP2), CCAAT/enhancer binding protein alpha $(C / E B P \alpha)$, adiponectin $(A D I P O Q)$ as well as oil red $\mathrm{O}$ staining of mature adipocytes and quantitative measurements of eluted oil red $\mathrm{O}$ as an indicator of the number of mature, lipid filled adipocytes (Additional file 5: Figure S4).

\section{In vivo heterotopic bone formation}

We examined the ability of hMSC-CD $146^{+}$and hMSC$\mathrm{CD}_{146^{-}}$populations to form heterotopic bone when implanted subcutaneously in immune-deficient mice together with HA/TCP. The CD146 status of the cells was confirmed by flow cytometry prior to implantation (Additional file 6: Figure S5). Bone formation was demonstrated in both hMSC-CD146 ${ }^{+}$and hMSC-CD $146^{-}$ (Fig. 4). Formation of BM within the implants was identified on the basis of the presence of large sinusoids (Fig. 4d-f) and BM elements (Fig. 4g-i, m). Quantification of bone formation demonstrated a significant difference between hMSC-CD146 ${ }^{-}(12.6 \pm 1.5)$ and hMSC$\mathrm{CD} 146^{+}(8.1 \pm 1.0)(P<0.05)$ (Fig. 4o). In contrast, significantly $(P<0.01)$ increased amounts of BM were identified in hMSC-CD146 ${ }^{+}(0.5 \% \pm 0.2 \%)$ as compared with hMSC-CD146- $(0.05 \pm 0.02)$ (Fig. 4p). Scans of whole implants demonstrating differences in bone formation between hMSC-CD146 ${ }^{+}$and hMSC-CD146 can be found in Additional file 7: Figure S6. Specificity of the vimentin staining was demonstrated by using murine
MSCs implanted under similar conditions to hMSCs (Additional file 8: Figure S7). Bone formed in hMSC implants was of human origin as evidenced by positive staining for a human-specific vimentin (Fig. 4j-1).

\section{In vitro and in vivo chemotaxis assay}

To explore other biological characteristics associated with the CD146 status, we examined the cells' ability for chemotaxis in vitro and in vivo. The hMSC-CD $146^{+}$ sorted population exhibited enhanced chemotactic responses to $10 \%$ FBS when compared with the $0.2 \%$ FBS control in an in vitro transwell migration assay. In contrast, hMSC-CD146- ${ }^{-}$cells demonstrated no significant migration between $0.2 \%$ (control) and $10 \%$ FBS (Fig. 5a). To examine the in vivo relevance of this observation, we injected LUC2-labelled $\mathrm{hMSC}-\mathrm{CD} 146^{+}$and hMSC-CD146- into the tail vein of immune-deficient mice with prior closed femur fracture. The mice were examined at d0, d3, and d6 (Fig. 5b). In all mice injected with hMSC-CD146-, the cells migrated to the lung, where the majority remained throughout the 6-day period. In contrast, hMSC-CD $146^{+}$cells were observed to migrate away from the lung area starting at day 3 and were recruited to both the injured right leg and noninjured left leg. By day 6, hMSC-CD $146^{+}$exhibited wider systemic distribution throughout the animals. Analysis of the bioluminescence radiance captured by using IVIS imaging demonstrated statistically significant differences between the lung and lower body radiance. Data from the lung radiance demonstrated a shift from significantly higher levels of hMSC-CD $146^{+}$radiance at $\mathrm{d} 0(P<0.05)$ to significantly lower radiance at day $6(P<0.01)$ as compared with hMSC-CD146 ${ }^{-}$. Analysis of the lower body demonstrated significantly increased radiance in the $\mathrm{hMSC}-\mathrm{CD} 146^{+}$at all time points $\left(* P<0.05,{ }^{* * *} P<0.01\right)$ as compared with hMSCCD146 radiance.

\section{Discussion}

Identification of prospective markers defining the functional ability of hMSCs is an important pre-requisite for use of hMSCs in therapy. In the present study, we employed CD146 to functionally phenotype BM hMSCs. Our results demonstrate that CD146 defines a population with bone-forming capacity as well as ability for in vivo trans-endothelial migration and homing to injured bone sites. 


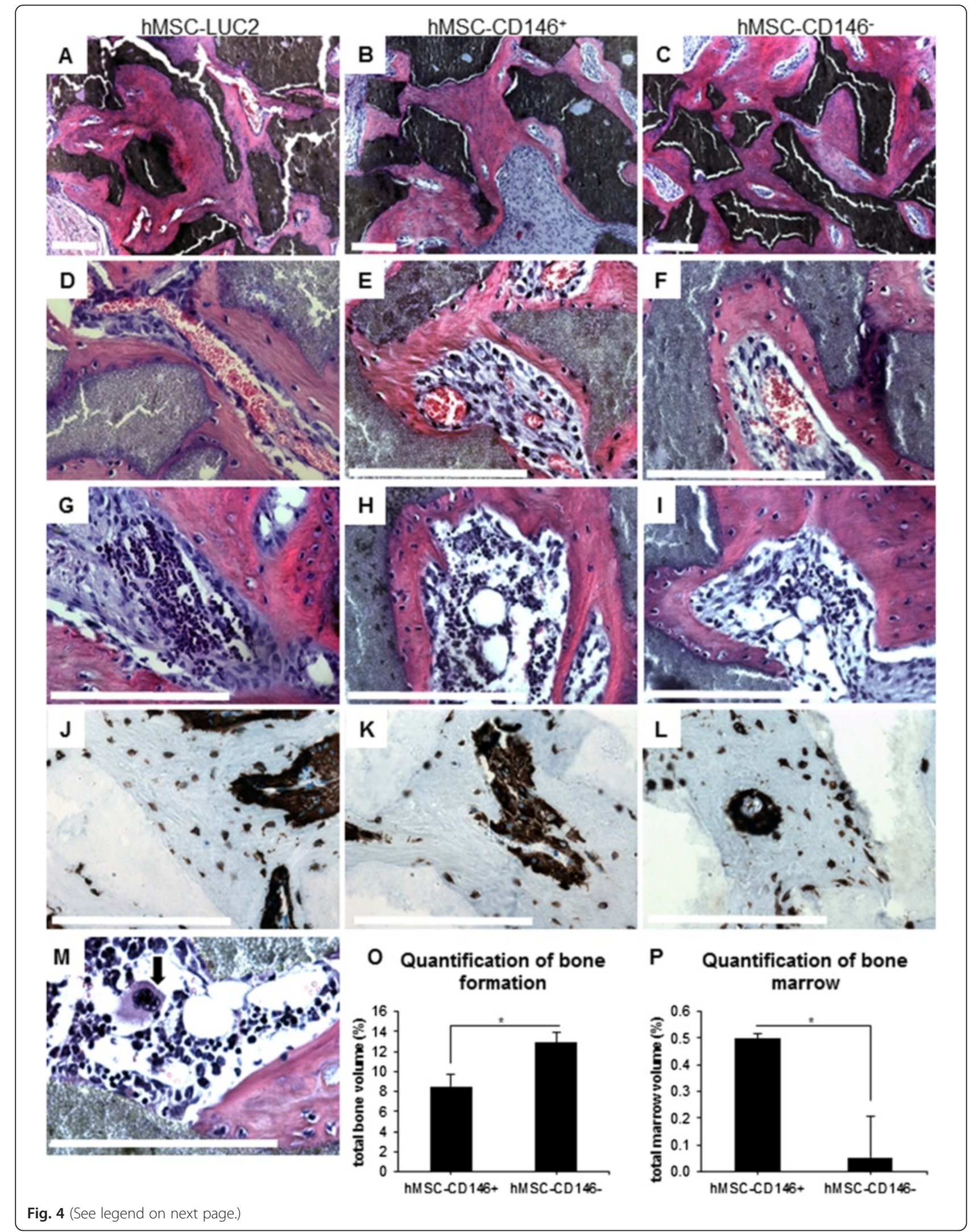


(See figure on previous page.)

Fig. 4 In vivo heterotopic bone formation. hMSC-LUC2 (a, d, g, j, m), hMSC-CD146 $(\mathbf{b}, \mathbf{e}, \mathbf{h}, \mathbf{k})$, and hMSC-CD146 $(\mathbf{c}, \mathbf{f}, \mathbf{i}, \mathbf{I})$ were implanted subcutaneously $(n=4)$ mixed with hydroxyapatite tricalcium phosphate/Triosite (HA/TCP) into immune-compromised mice for 8 weeks. Analysis was performed on three serial sections at three depths with $100 \mu \mathrm{m}$ between each depth. Hematoxylin and eosin (H\&E) staining of bone formation demonstrating distribution of bone within the implants $(\mathbf{a}-\mathbf{i}, \mathbf{m})$. $\mathbf{d}-\mathbf{f}$ Blood vessel formation within the implants. $\mathbf{g}$-i Establishment of bone marrow within the implants. $\mathbf{j}-\mathbf{l}$ Human-specific vimentin staining of developing bone demonstrating the human origin. $\mathbf{o}, \mathbf{p}$ Quantification of total bone or bone marrow volume. Scale bar on all H\&E and vimentin staining $=100 \mu \mathrm{m}$

Previous studies have suggested a number of biological roles for CD146, including trans-endothelial migration [24-27], cell proliferation regulation [27-29], and a role in cancer metastases [30-32]. In addition, CD146 has also been proposed as a pericyte cell marker [33, 34] and as a cell marker for hMSCs and osteoblastic cells $[27,33,35]$. Here, we report that CD146 status is associated with progenitor functions of hMSCs which include differentiation into osteoblasts and adipocytes as well as ability for trans-endothelial migrations in vivo.

Both hMSC-CD146 ${ }^{+}$and hMSC-CD146 ${ }^{-}$cells were able to differentiate readily into osteoblastic and adipocytic cells with similar efficiency, and both populations formed heterotopic bone and BM when implanted in immune-deficient mice. Our results corroborate previous results that also demonstrated, in primary BM hMSCs, that CD146 status did not influence response to differentiation induction to osteoblasts, adipocytes, or chondrocytes [28]. Recently, Gothard et al. compared $\mathrm{CD}_{146^{+}}$cells with Stro1 ${ }^{+}$and demonstrated similar gene expression profiles [13]. Similarly, Russell et al. reported that $\mathrm{CD} 146^{+}$cells are enriched in multipotent hMSCs [7]. Although these studies did conduct side-by-side comparison of $\mathrm{CD}_{146^{+}}$and $\mathrm{CD} 146^{-} \mathrm{hMSC}$ populations, the current data suggest that $\mathrm{CD} 146^{+}$status defines the progenitor cell population of hMSCs.

Morphological changes of the cytoskeleton, including reorganization of actin fibres, are important for osteoblast cell differentiation [36]. Recent data have demonstrated an increase in the density of F-actin during hMSC differentiation [37]. Using the SER features from the Harmony software, we analyzed the cytoplasmic texture and demonstrated significant differences in the sizes of the F-actin fibres between hMSC-CD146 ${ }^{+}$and hMSC$\mathrm{CD} 146^{-}$cells, and actin fibres were significantly larger in the hMSC-CD146 ${ }^{-}$population. Sonowal et al. [38], McBeath et al. [39], and Treiser et al. [40] have reported an association between actin fibre and cytoskeletal modification with osteoblastic differentiation. Thus, the observed changes in actin fibres in $\mathrm{CD}_{146^{-}}$cells may contribute to their more mature $\mathrm{OB}$ phenotype.

We identified that CD146 defines cells with ability for trans-endothelial migration. Statistically significant data demonstrated that hMSC-CD $146^{+}$cells migrated away from the lungs; however, homing to a site of injury was not consistently observed. Consistent with these findings, we observed that the hMSC-CD146 ${ }^{+}$cells exhibited a smaller cell size and cytoskeletal morphology, suggesting enhanced motility. Our results corroborate results from previous investigators that CD146 confers additional functional phenotype on hMSCs. Espangolle et al. reported that $\mathrm{CD} 146^{+}$cells exhibited an enriched vascular smooth muscle cell phenotype [28]. Ye et al. [26] demonstrated that CD146 acts as a receptor for Wnt5A, mediating induced cell migration. Along similar lines to our study, Blocki et al. [33] reported that although both CD $146^{+}$and CD146 ${ }^{-}$populations of BM hMSCs exhibited an equivalent differentiation ability for adipogenic and osteoblastic lineages, only CD146 ${ }^{+}$cells formed endothelial tubular networks on Matrigel ${ }^{\mathrm{m}}$. The endothelial-like phenotype may be related to trans-endothelial migration ability.

We identified a significant difference between the amount of bone formed by hMSC-CD146 ${ }^{+}$and hMSCCD146- cells. hMSC-CD146- formed more bone but demonstrated a 10-fold decrease in BM compared with hMSC-CD146 ${ }^{+}$. hMSC-CD146 ${ }^{-}$maintained their CD146status throughout the experiments, but hMSC-CD146 cells were observed to be returning to a phenotypic equilibrium after six passages post-cell sort. Thus, it is possible that utilization of cells where $100 \%$ were positive for CD146 may enhance the quantity of BM and decrease the amount of bone formed. Previous reports have demonstrated that $\mathrm{CD} 146^{+}$hMSCs are located in the perivascular spaces [14] and have been associated with reestablishment of the haematopoietic microenvironment [18]. In comparison, CD146 ${ }^{-}$cells were found to line the bone cavity [14]. The different locations of $\mathrm{CD} 146^{+}$or $\mathrm{CD} 146^{-}$cells could suggest that, within the heterogeneous MSC population, cells have different degrees of maturity: $\mathrm{CD}_{146^{-}}$form more bone and less marrow and are thus more mature, whereas $\mathrm{CD} 146^{+}$retain more plasticity (that is, in addition to bone formation and BM formation, they have a trans-endothelial migration capacity).

\section{Conclusions}

We hypothesize that $\mathrm{CD} 146^{+}$cells are recruited to bone surfaces by trans-endothelial migration [41]. Further maturation to active osteoblasts is associated with loss of CD146 and thus cells located on active bone-forming surfaces are $\mathrm{CD} 146^{-}$. Changes in the expression of CD146 reveal a dual function in hMSC biology: a role 


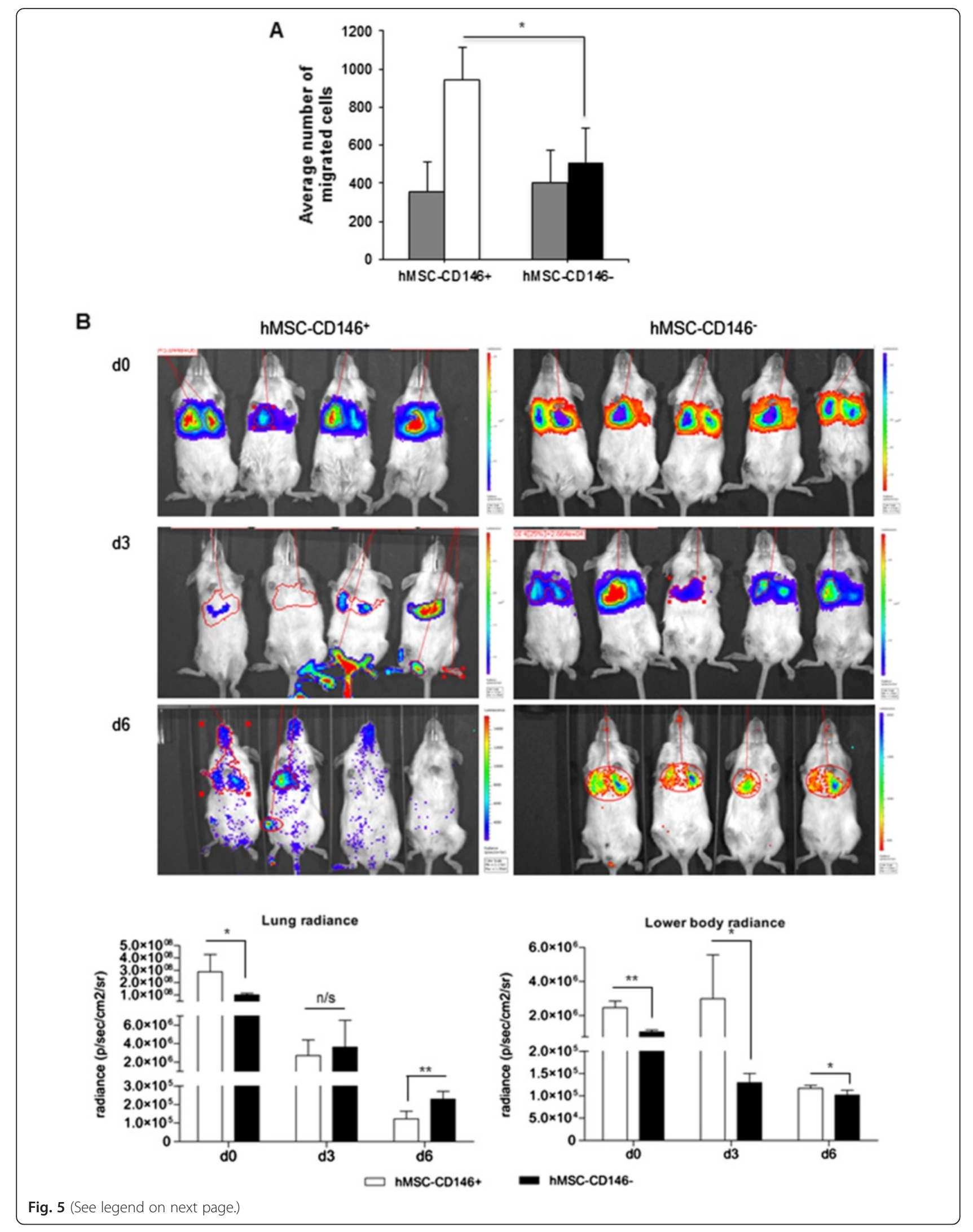


(See figure on previous page.)

Fig. 5 Functional assessment of migrational ability of CD146 sorted human mesenchymal stem cell (hMSC) populations. a Analysis of in vitro transwell (Boyden chamber) migration assay. Data demonstrate statistically significant migration of hMSC-CD146 ${ }^{+}$in $10 \%$ fetal bovine serum

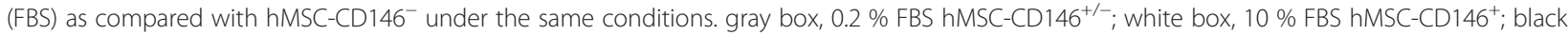
box, $10 \%$ FBS hMSC-CD146, $\mathrm{n}=2$ independent experiments. ${ }^{*} P<0.05 . \mathbf{b} \mathrm{IVIS}{ }^{\circ}$ in vivo imaging of hMSC-CD $146^{+}$and $\mathrm{hMSC}-\mathrm{CD} 146^{-}$cells at days 0 , 3 , and 6 after intravenous tail vein injection. As can be observed in the images and from the radiance plots, hMSC-CD146 ${ }^{+}$cells ( $n=4$ mice) demonstrate migration away from the lung area at days 3 and 6 , and hMSC-CD146- cells ( $n=5$ mice) display less migration in comparison with hMSC-CD146

in enhancing migration and recruitment to bone surfaces $\left(\mathrm{CD} 146^{+}\right)$and a role in osteoblastic commitment and bone formation $\left(\mathrm{CD} 146^{-}\right)$. Further studies examining molecular details of mechanisms underlying these two functions need to be investigated.

\section{Additional files}

Additional file 1: Table S1. Sequence data for primers used within these studies. (DOCX $13 \mathrm{~kb}$ )

Additional file 2: Figure S1. Characterization of hMSC-LUC2 as compared with the parental cell line hMSC-TERT. A Morphology. B Long-term growth rate. ${ }^{*} P<0.05$. C Flow cytometric analysis of CD marker expression. Scale bar $=500$ or $100 \mu \mathrm{m}$. hMSC human mesenchymal stem cell. (TIF $423 \mathrm{~kb}$ )

Additional file 3: Figure S2. Characterization of osteoblast and adipocyte differentiation capacity of hMSC-TERT and hMSC-LUC2. A Osteoblastic differentiation. B Adipocytic differentiation. ${ }^{*} P<0.05$, ${ }^{* *} P<0.005$, ${ }^{* *} P<0.001$; scale bar $=100 \mu \mathrm{m}$ : black box, hMSC-TERT; white box, hMSC-LUC2. ALP alkaline phosphatase, AZR Alizarin red, hMSC human mesenchymal stem cell. (TIF $522 \mathrm{~kb}$ )

Additional file 4: Figure S3. Characterization and comparison of in vivo bone formation of hMSC-TERT and hMSC-LUC2. A, B Scanned images of hMSC-TERT and hMSC-LUC2 implants. Hematoxylin-and-eosin staining of hMSC-TERT (C) and hMSC-LUC2 (D). E hMSC-LUC2 implants: humanspecific vimentin staining demonstrating the human origin of the cells. Images of chondrocyte (F), blood vessel (G), and osteoclast (H). Scale bar $=100 \mu \mathrm{m}$. hMSC human mesenchymal stem cell. (TIF $1294 \mathrm{~kb}$ )

Additional file 5: Figure S4. In vitro adipocytic differentiation of hMSC-CD146 ${ }^{+}$and hMSC-CD146- cell populations. A Oil red O staining. B Reverse transcription-polymerase chain reaction analysis of adipocytic gene expression. $n=3$ independent experiments, mean \pm standard error of the mean; white box, hMSC-CD146 ${ }^{+}$; black box, hMSC-CD146-; scale bar $=100 \mu \mathrm{m}$. hMSC human mesenchymal stem cell. (TIF $505 \mathrm{~kb}$ )

Additional file 6: Figure S5. CD profile of hMSC-TERT, hMSC-CD146+, and hMSC-CD146- populations prior to in vivo implantation. hMSC human mesenchymal stem cell. (TIF $150 \mathrm{~kb}$ )

Additional file 7: Figure S6. Scanned images (hematoxylin and eosin and human-specific vimentin) of whole implants of hMSC-CD146 ${ }^{+}$and hMSC-CD146 ${ }^{-}$cells implanted into immune-compromised mice for 8 weeks. Cells of murine origin can be observed in the non-stained areas of implants as demonstrated by arrows. hMSC human mesenchymal stem cell. (TIF $1385 \mathrm{~kb})$

Additional file 8: Figure S7. Validation of human-specific vimentin staining. A, B Human mesenchymal stem cells (hMSCs) implanted on hydroxyapatite tricalcium phosphate/Triosite (HA/TCP) in immunecompromised mice. C, D Murine mesenchymal stem cells (mMSCs) implanted on HA/TCP in immune-compromised mice. No vimentin staining can be observed in murine cells. Scale bar $=100 \mu \mathrm{m}$. (TIF $1979 \mathrm{~kb})$

\section{Abbreviations}

ALP: Alkaline phosphatase: BM: Bone marrow; BSA: Bovine serum albumin; CD146: Cluster of differentiation 146; CT: Cycle threshold;
EDTA: Ethylenediaminetetraacetic acid; FACS: Fluorescence-activated cell sorting; FBS: Fetal bovine serum; HATCP: Hydroxyapatite tricalcium phosphate/Triosite; hMSC: Human skeletal (stromal) mesenchymal stem cell; IgCD146: Long form of CD146; LUC2: Luciferase; MSC: Mesenchymal stem cell; ORO: Oil red O; PBS: Phosphate-buffered saline; PCR: Polymerase chain reaction; RT-PCR: Reverse transcription-polymerase chain reaction; SER: Saddles, edges, ridges; shCD146: Short form of CD146.

\section{Competing interests}

The authors declare that they have no competing interests.

\section{Authors' contributions}

LH was responsible for the experimental concept, design, and manuscript preparation and performed routine cell culture, population doubling, flow cytometry, RT-PCR, ALP activity, cytochemical and fluorescent staining, high-content imaging and analysis, bone quantification, and manuscript preparation. MK was responsible for the experimental concept, design, and manuscript preparation. ND carried out implantation experiments, microcomputed tomography imaging, fractures, and IVIS imaging and analysis. WZ performed cell sorting and in vivo tail vein injections and IVIS imaging. Al performed in vitro migration assays and analysis. All authors critically read for intellectual content, approved the manuscript, and contributed to ongoing experimental design and discussion during experimentation

\section{Acknowledgments}

The authors wish to thank Weimin Qiu and Kenneth Hauberg Larsen for helping in creation of the hMSC-LUC2 cell line and Lone Christiansen and Tina K. Nielsen for excellent technical assistance. This work was supported by grants from the Danish Strategic Research Council (09-067112), Novo Nordisk Foundation (2014-10309), and the University Hospital of Odense, Odense, Denmark.

\section{Author details}

'Department of Endocrinology and Metabolism, Molecular Endocrinology Laboratory (KMEB), Odense University Hospital, University of Southern Denmark, Winslowparken 25.1, 5000 Odense C, Denmark. ${ }^{2}$ Danish Stem Cell Center (DanStem), Panum Institute, University of Copenhagen, Blegdamsvej 3B, Copenhagen 2200, Denmark. 'Stem Cell Unit, Department of Anatomy, College of Medicine, King Saud University, 4852 Ash Shaikh Hasan Ibn Abdullah Al Ash Shaikh, Riyadh 11461, Kingdom of Saudi Arabia.

Received: 18 August 2015 Revised: 25 November 2015 Accepted: 17 December 2015

Published online: 11 January 2016

\section{References}

1. Abdallah BM, Kassem M. New factors controlling the balance between osteoblastogenesis and adipogenesis. Bone. 2012;50:540-5.

2. Quarto R, Mastrogiacomo M, Cancedda R, Kutepov SM, Mukhachev V, Lavroukov A, et al. Repair of large bone defects with the use of autologous bone marrow stromal cells. N Engl J Med. 2001;344:385-6.

3. Murphy MB, Moncivais K, Caplan Al. Mesenchymal stem cells: environmentally responsive therapeutics for regenerative medicine. Exp Mol Med. 2013;45:e54

4 Wakitani S, Imoto K, Yamamoto T, Saito M. Murata N, Yoneda M. Human autologous culture expanded bone marrow mesenchymal cell transplantation for repair of cartilage defects in osteoarthritic knees. Osteoarthr Cartil. 2002;10:199-206. 
5. Kuznetsov SA, Krebsbach PH, Satomura K, Kerr J, Riminucci M, Benayahu D, et al. Single-colony derived strains of human marrow stromal fibroblasts form bone after transplantation in vivo. J Bone Miner Res. 1997;12:1335-47.

6. Muraglia A, Cancedda R, Quarto R. Clonal mesenchymal progenitors from human bone marrow differentiate in vitro according to a hierarchical model. J Cell Sci. 2000;113:1161-6.

7. Russell KC, Phinney DG, Lacey MR, Barrilleaux BL, Meyertholen KE, O'Connor KC. In vitro high-capacity assay to quantify the clonal heterogeneity in trilineage potential of mesenchymal stem cells reveals a complex hierarchy of lineage commitment. Stem Cells. 2010;28:788-98.

8. Larsen KH, Frederiksen CM, Burns JS, Abdallah BM, Kassem M. Identifying a molecular phenotype for bone marrow stromal cells with in vivo bone-forming capacity. J Bone Miner Res. 2010;25:796-808.

9. Post S, Abdallah BM, Bentzon JF, Kassem M. Demonstration of the presence of independent pre-osteoblastic and pre-adipocytic cell populations in bone marrow-derived mesenchymal stem cells. Bone. 2008;43:32-9.

10. Kassem M, Bianco P. Skeletal stem cells in space and time. Cell. 2015;160: 17-9.

11. Phinney DG. Biochemical heterogeneity of mesenchymal stem cell populations: clues to their therapeutic efficacy. Cell Cycle. 2007;6:2884-9.

12. Phinney DG. Functional heterogeneity of mesenchymal stem cells: Implications for cell therapy. J Cell Biochem. 2012;113:2806-12.

13. Gothard D, Greenhough J, Ralph E, Oreffo RO. Prospective isolation of human bone marrow stromal cell subsets: a comparative study between Stro-1-, CD146- and CD105-enriched populations. J Tissue Eng. 2014;5: 2041731414551763.

14. Tormin A, Li O, Brune JC, Walsh S, Schütz B, Ehinger M, et al. CD146 expression on primary nonhematopoietic bone marrow stem cells is correlated with in situ localization. Blood. 2011;117:5067-77.

15. Battula VL, Treml S, Bareiss PM, Gieseke F, Roelofs $H$, de Zwart P, et al. Isolation of functionally distinct mesenchymal stem cell subsets using antibodies against CD56, CD271, and mesenchymal stem cell antigen-1. Haematologica. 2009;94:173-84.

16. Buhring HJ, Treml S, Cerabona F, de Zwart P, Kanz L, Sobiesiak M. Phenotypic characterization of distinct human bone marrow-derived MSC subsets. Ann N Y Acad Sci. 2009:1176:124-34.

17. Bianco $P$, Costantini M, Dearden LC, Bonucci E. Alkaline phosphatase positive precursors of adipocytes in the human bone marrow. $\mathrm{Br} J$ Haematol. 1988;68:401-3.

18. Sacchetti B, Funari A, Michienzi S, Di Cesare S, Piersanti S, Saggio I, et al. Self-renewing osteoprogenitors in bone marrow sinusoids can organize a hematopoietic microenvironment. Cell. 2007;131:324-36.

19. Ulrich C, Abruzzese T, Maerz JK, Ruh M, Amend B, Benz K, et al. Human placenta-derived CD146-positive mesenchymal stromal cells display a distinct osteogenic differentiation potential. Stem Cells Dev. 2015;24:1558-69.

20. Simonsen JL, Rosada C, Serakinci N, Justesen J, Stenderup K, Rattan SIS, et al. Telomerase expression extends the proliferative life-span and maintains the osteogenic potential of human bone marrow stromal cells. Nat Biotech. 2002:20:592-6.

21. Harkness L, Mahmood A, Ditzel N, Abdallah BM, Nygaard JV, Kassem M. Selective isolation and differentiation of a stromal population of human embryonic stem cells with osteogenic potential. Bone. 2011;48:231-41.

22. Bonnarens F, Einhorn TA. Production of a standard closed fracture in laboratory animal bone. J Orthop Res. 1984;2:97-101.

23. Kebir A, Harhouri K, Guillet B, Liu JW, Foucault-Bertaud A, Lamy E, et al. CD146 short isoform increases the proangiogenic potential of endothelial progenitor cells in vitro and in vivo. Circ Res. 2010;107:66-75.

24. Kim SJ, Kim SY, Kwon CH, Kim YK. Differential effect of FGF and PDGF on cell proliferation and migration in osteoblastic cells. Growth Factors. 2007; 25:77-86.

25. Shinojima N, Hossain A, Takezaki T, Fueyo J, Gumin J, Gao F, et al. TGF-beta mediates homing of bone marrow-derived human mesenchymal stem cells to glioma stem cells. Cancer Res. 2013;73:2333-44.

26. Ye Z, Zhang C, Tu T, Sun M, Liu D, Lu D, et al. Wnt5a uses CD146 as a receptor to regulate cell motility and convergent extension. Nat Commun. 2013;4:2803.

27. Zhu W, Tan Y, Qiu Q, Li X, Huang Z, Fu Y, et al. Comparison of the properties of human CD146+ and CD146- periodontal ligament cells in response to stimulation with tumour necrosis factor alpha. Arch Oral Biol. 2013;58:1791-803.
28. Espagnolle N, Guilloton F, Deschaseaux F, Gadelorge M, Sensébé L, Bourin P. CD146 expression on mesenchymal stem cells is associated with thei vascular smooth muscle commitment. J Cell Mol Med. 2014;18:104-14.

29. Maijenburg MW, Kleijer M, Vermeul K, Mul EPJ, van Alphen FPJ, van der Schoot CE, et al. The composition of the mesenchymal stromal cell compartment in human bone marrow changes during development and aging. Haematologica. 2012;97:179-83.

30. Luo Y, Zheng C, Zhang J, Lu D, Zhuang J, Xing S, et al. Recognition of CD146 as an ERM-binding protein offers novel mechanisms for melanoma cell migration. Oncogene. 2012;31:306-21.

31. Zeng G, Cai S, Liu Y, Wu GJ. METCAM/MUC18 augments migration, invasion, and tumorigenicity of human breast cancer SK-BR-3 cells. Gene. 2012;492: 229-38.

32. Imbert AM, Garulli C, Choquet E, Koubi M, Aurrand-Lions M, Chabannon C. CD146 expression in human breast cancer cell lines induces phenotypic and functional changes observed in epithelial to mesenchymal transition. PLoS One. 2012;7:e43752.

33. Blocki $A$, Wang $Y$, Koch $M$, Peh $P$, Beyer $S$, Law $P$, et al. Not all MSCs can act as pericytes: functional in vitro assays to distinguish pericytes from other mesenchymal stem cells in angiogenesis. Stem Cells Dev. 2013;22:2347-55.

34. Li Q, Yu Y, Bischoff J, Mulliken JB, Olsen BR. Differential expression of CD146 in tissues and endothelial cells derived from infantile haemangioma and normal human skin. J Pathol. 2003;201:296-302.

35. Tsang WP, Shu Y, Kwok PL, Zhang F, Lee KK, Tang MK, et al. CD146+ human umbilical cord perivascular cells maintain stemness under hypoxia and as a cell source for skeletal regeneration. PLoS One. 2013;8:e76153.

36. Yourek G, Hussain MA, Mao JJ. Cytoskeletal changes of mesenchymal stem cells during differentiation. ASAIO J. 2007:53:219-28.

37. Sliogeryte K, Thorpe SD, Lee DA, Botto L, Knight MM. Stem cell differentiation increases membrane-actin adhesion regulating cell blebability, migration and mechanics. Sci Rep. 2014;4:7307.

38. Sonowal H, Kumar A, Bhattacharyya J, Gogoi PK, Jaganathan BG. Inhibition of actin polymerization decreases osteogeneic differentiation of mesenchymal stem cells through p38 MAPK pathway. J Biomed Sci. 2013; 20:71.

39. McBeath R, Pirone DM, Nelson CM, Bhadriraju K, Chen CS. Cell shape, cytoskeletal tension, and RhoA regulate stem cell lineage commitment. Dev Cell. 2004;6:483-95.

40. Treiser MD, Yang EH, Gordonov S, Cohen DM, Androulakis IP, Kohn J, et al. Cytoskeleton-based forecasting of stem cell lineage fates. Proc Natl Acad Sci U S A. 2010;107:610-5.

41. Delaisse JM. The reversal phase of the bone-remodeling cycle: cellular prerequisites for coupling resorption and formation. Bonekey Rep. 2014;3: 561.

\section{Submit your next manuscript to BioMed Central and we will help you at every step:}

- We accept pre-submission inquiries

- Our selector tool helps you to find the most relevant journal

- We provide round the clock customer support

- Convenient online submission

- Thorough peer review

- Inclusion in PubMed and all major indexing services

- Maximum visibility for your research

Submit your manuscript at www.biomedcentral.com/submit
C Biomed Central 